\title{
Buckling and Vibration Analysis of Functionally Graded Composite Structures using the Finite Element Method
}

\author{
O.O. Oyekoya ${ }^{2}$, D.U. Mba $^{1} \&$ A.M. El-Zafrany \\ 1 \& 2 Department of Structures Impact and Machine Dynamics, School of Engineering, Cranfield University, Cranfield, \\ Bedfordshire, MK43 0AL, England \\ ${ }^{1}$ Email: d.u.mba@cranfield.ac.uk
}

\begin{abstract}
The authors [1] have previously written a paper on structural integrity of functionally graded composite (FGC) structure using Mindlin-type finite elements. In this paper, the Mindlin-type element and Reissner-type element have been further developed for the modelling of FGC plate subjected to buckling and free vibration. The Mindlin-type element formulation is based on averaging of transverse shear distribution over plate thickness using Lagrangian interpolation. The Reissner-type element formulation is based on parabolic transverse shear distribution over plate thickness using Lagrangian and Hermitian interpolation. The composite plate considered in this paper is functionally graded in the longitudinal direction only, but the FE code developed is capable of analysing composite plates with functional gradation in transverse and radial direction as well. This study was able to show that the structural integrity enhancement and strength maximisation of composite structures are achievable through functional gradation of material properties over the structure.
\end{abstract}

KEY WORDS: Functionally graded material, finite element methods, Mindlin-type element, Reissner-type element, finite strain, progressive damage analysis 


\section{List of Symbols}

K

$\mathbf{R}$

$\delta$

$\tau$

$\varepsilon$

$\vec{a}$

$\mathrm{F}_{\mathrm{i}}, \mathrm{G}_{\mathrm{i}}$

$\mathrm{H}_{\mathrm{i}}, \mathrm{P}_{\mathrm{i}} \quad$ Hermitian shape functions

$\mathrm{N}_{\mathrm{i}} \quad$ Lagrangian shape functions

U Strain energy

$\mathrm{u}, \mathrm{v}, \mathrm{w}$

Displacement components along the $\mathrm{x}, \mathrm{y}$

and $\mathrm{z}$ directions respectively

V Volume fraction

W Work done by actual load

X Longitudinal strength

Y Transverse strength

$\xi, \eta \quad$ Non-dimensional $\mathrm{x}$ and $\mathrm{y}$ location

$\rho \quad$ Density

$\mu \quad$ Shear modulus

$\psi \quad$ Transverse shear deformation

$v \quad$ Poisson ratio
( ) $\quad$ Longitudinal direction of the material axis

( ) 2 Transverse direction of the material axis

( ) $\quad$ Compressive strength

( ) Fibre

( ) $\quad$ Matrix

( ) Tensile strength

( ) Longitudinal direction of the local axis

( ) Transverse direction of the local axis

( ) comp Traditional composite

( ) $\quad$ fgunctionally graded material

( ) $\quad$ Out of plane displacement component

( ) $\quad \mathrm{u}$ and $\mathrm{v}$ displacement components

( ) w displacement component

( ) Midplane displacement component

( ) $\quad$ Transverse shear component

( ) $\quad$ Bending component

()$^{\mathrm{L}} \quad$ Lth layer of composite

( ) $\quad$ Midplane displacement component

()$^{\sigma} \quad$ Non-linear terms

\section{Introduction}

Functionally Graded Materials (FGMs) are made such that the volume fractions of two or more materials are varied continuously along a certain dimension. The FGM concept originated in Japan in 1984 during the spaceplane project, in the form of a proposed thermal barrier material capable of withstanding a surface temperature of 
$2000 \mathrm{~K}$ and a temperature gradient of $1000 \mathrm{~K}$ across a cross section $<10 \mathrm{~mm}$ [2]. In 2000, Reddy [3] presented a theoretical formulation and finite element models based on third order shear deformation theory for the analysis of through-thickness functionally graded plates. The Navier solution for simply supported plates based on the linear third-order theory and the non-linear static and dynamic finite element results based on the first-order theory were presented by Reddy in [3]. The results show the effects of volume fractions and modulus ratio of the constituents on deflections and transverse shear stresses. In 2002, Javaheri et al. [4] derived equilibrium and stability equations for rectangular simply supported functionally graded plates. Javaheri's derivation was based on the classical plate theory with the assumption of power law composition for the material and he studied the buckling analysis of functionally graded plates under in-plane compression. In 2004, Chen et al. [2] investigated the buckling behaviour of FGM rectangular plates subjected to non-linearly distributed in-plane edge loads. Chen et al. [2] stated that a mesh-free method which approximates displacements based on scattered nodes (i.e. radial basis function and polynomial basis) was employed, in-order to avoid complicated numerical procedures that arises in the FEM from the use of elements. This FEM complication was dealt with in this paper. Other useful studies on functionally graded materials can be found in these references [5 - 11].
In comparison to existing publications, this paper has been able to give unique contributions to the subject matter. These contributions include Mindlin-type element formulation, Reissner-type element formulation, finite strain modelling and smooth fibre distribution technique. The Mindlin-type element formulation is based on an assumption of average transverse shear distribution over plate thickness using Lagrangian interpolation. The Reissner-type element formulation is based on an assumption of parabolic transverse shear distribution over plate thickness using Lagrangian and Hermitian interpolation. Green's strain-displacement equation was employed in the finite strain modelling. The smooth fibre distribution technique is based on the numerical computation of macro-mechanical properties at Gaussian quadrature points.

The authors [1] have previously written a paper on structural integrity of functionally graded composite structure using Mindlin-type finite elements. In the paper [1], two new Mindlin-type plate bending elements were derived for the modelling of functionally graded plate subjected to various loading conditions such as tensile loading, in-plane bending and out-of-plane bending. There were two types of non-linearity considered in the modelling of the plate, which include finite strain and material degradation. In the Mindlin-type element formulation, the transverse shear strain is averaged over 
plate thickness. Its finite element derivation is based on Lagrangian interpolation.

In order to model the transverse shear strain more accurately, the Reissner-type element is derived in this paper, which is based on parabolic distribution of transverse shear strain over plate thickness. Its finite element derivation is complex because it is based on Lagrangian and Hermitian interpolations.

In this paper, the Mindlin-type element and Reissnertype element have been further developed for the modelling of functionally graded composite plate subjected to buckling and free vibration. Vibration and buckling analysis were then undertaken for different fibre distribution cases and the effects of fibre distribution were studied. Fibre distribution cases with maximum vibration frequency and maximum buckling loads were chosen as the optimum design.

\subsection{Optimisation Technique}

The optimisation technique used in this paper can be described as a fail-safe design technique which involves the imposition of constraints to ensure that the physical limitations of materials or structural properties required for satisfactory performance are not exceeded. This optimisation technique involves changing the fibre distribution parameters and running the FE code for the given fibre distribution, checking to see if all constraints have been satisfied. The constraints that have been considered include buckling load constraints and natural frequency constraints. The flow chart below is a good description of this optimisation technique concept.

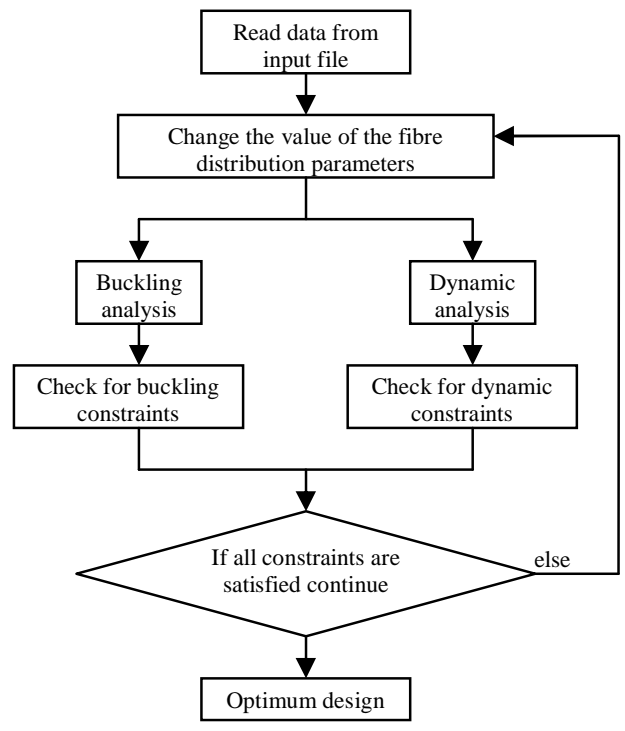

Figure 1: Optimisation technique

\section{Micro-mechanics of Fibrous Composites}

This section defines the elastic and strength properties of FGMs [12]. It also describes the micromechanics algorithm and the fibre distribution techniques such as average and smooth fibre distribution technique.

\subsection{Elastic Properties of FGMs}

The longitudinal stiffness of a composite can be obtained from the rule or law of mixture which is represented by the relationship given below.

$$
\mathrm{E}_{11}=\mathrm{E}_{\mathrm{f}} \mathrm{V}_{\mathrm{f}}+\mathrm{E}_{\mathrm{m}} \mathrm{V}_{\mathrm{m}}
$$

The transverse stiffness of a composite is given below. 


$$
\frac{1}{E_{22}}=\frac{V_{f}}{E_{f}}+\frac{V_{m}}{E_{m}}
$$

The in-plane shear modulus can be obtained from a similar model to that used for obtaining transverse stiffness which results in the in-plane shear modulus expression as given below.

$$
\frac{1}{\mu_{12}}=\frac{V_{f}}{\mu_{f}}+\frac{V_{m}}{\mu_{m}}
$$

The Poisson's ratio of a composite is given below.

$$
v_{12}=V_{f} v_{f}+V_{m} v_{m}
$$

\subsection{Strength Properties of FGMs}

The longitudinal tensile strength of FGMs is given below.

$$
\begin{aligned}
& X_{t}=X_{f t}\left[V_{f}+V_{m} \frac{E_{m}}{E_{1 f}}\right] \\
& X_{t}=X_{m t}\left[V_{m}+V_{f} \frac{E_{1 f}}{E_{m}}\right]
\end{aligned}
$$

The longitudinal compressive strength of FGMs for $V_{f}<$ 0.5 is given below.

$$
\begin{aligned}
& X_{c}=2 V_{f}\left[\frac{E_{m} E_{1 f} V_{f}}{3\left(1-v_{f}\right)}\right] \\
& X_{c}=2 V_{f}\left[\frac{E_{m} E_{1 f} V_{f}}{3 v_{m}}\right]
\end{aligned}
$$

$$
X_{c}=2 X_{f s}\left[V_{f}+V_{m} \frac{E_{m}}{E_{1 f}}\right]
$$

The transverse tensile strength of FGMs is given below

$$
\mathrm{Y}_{\mathrm{t}}=\frac{\mathrm{X}_{\mathrm{mt}}-\sigma_{\mathrm{rm}}}{\mathrm{k}_{\mathrm{c}}}
$$

The transverse compressive strength of FGMs is given below.

$$
\mathrm{Y}_{\mathrm{c}}=\frac{\mathrm{X}_{\mathrm{mc}}+\sigma_{\mathrm{rm}}}{\mathrm{k}_{\mathrm{c}}}
$$

The shear strength of FGMs is given below.

$$
\mathrm{S}=\frac{\mathrm{X}_{\mathrm{ms}}}{\mathrm{k}_{\mathrm{s}}}
$$

\subsection{Fibre Distribution Techniques}

This section explains the implementation of fibre distribution in the FE code. The equation used for fibre distribution is given below.

$$
V_{f}(\xi)=V_{1}+\left(V_{2}-V_{1}\right) \xi^{p}
$$

where

$$
\begin{aligned}
& \xi=\frac{x-x_{1}}{x_{2}-x_{1}}, \quad \text { for } i c=1 \\
& \xi=\frac{y-y_{1}}{y_{2}-y_{1}}, \quad \text { for } i c=2 \\
& \xi=\frac{r-r_{1}}{r_{2}-r_{1}}, \quad \text { for } i c=3 \\
& V_{1}=\text { fibre ratio at } x_{1}, y_{1} \text { or } r_{1} \\
& V_{2}=\text { fibre ratio at } x_{2}, y_{2} \text { or } r_{2} \\
& p=\text { power index }
\end{aligned}
$$




\section{$\underline{\text { Manual Fibre Distribution }}$}

This fibre distribution technique enables the code user to manually specify the fibre ratio for each element along the direction of fibre ratio variation.

\section{Average Fibre Distribution}

This fibre distribution technique enables the code user to either specify fibre ratios $V_{1}$ and $V_{2}$ or the mean fibre ratio $\overline{\mathrm{V}}$ and fibre ratio $\mathrm{V}_{1}$. These fibre ratios are then used in computing the fibre ratios at the midpoint of each element using equations which will be derived later in this section.

\section{$\underline{\text { Smooth Fibre Distribution }}$}

This fibre distribution technique enables the code user to either specify fibre ratios $V_{1}$ and $V_{2}$ or the mean fibre ratio $\overline{\mathrm{V}}$ and fibre ratio $\mathrm{V}_{1}$. These fibre ratios are then used in computing the fibre ratios at each Gaussian quadrature point using equations which will be derived later in this section.

$\underline{\text { Derivation of mean fibre ratio }} \underline{\overline{\mathrm{V}}} \underline{\text { for specified fibre ratios }}$ $\underline{\mathrm{V}}_{1}$ and $\mathrm{V}_{2}$

Using the fibre distribution equation, the mean fibre ratio can be written as shown below.

$$
\overline{\mathrm{V}}_{\mathrm{f}}(\xi)=\frac{\int_{\xi_{\min } \mathrm{V}(\xi) \mathrm{d} \xi}^{\xi_{\max }}}{\xi_{\max }-\xi_{\min }}
$$

Hence

$$
\overline{\mathrm{V}}_{\mathrm{f}}(\xi)=\frac{\mathrm{V}_{1}\left(\xi_{\text {max }}-\xi_{\text {min }}\right)+\frac{\left(\mathrm{V}_{2}-\mathrm{V}_{1}\right)\left(\xi_{\text {max }}^{\mathrm{p}+1}-\xi_{\text {min }}^{\mathrm{p}+1}\right)}{\mathrm{p}+1}}{\xi_{\text {max }}-\xi_{\text {min }}}
$$

Derivation of fibre ratio at both ends of plate $\left(\mathrm{V}_{1} \& \mathrm{~V}_{2}\right)$ $\underline{\text { and mean fibre ratio }}$

Rearranging the equation below,

$$
\overline{\mathrm{V}}_{\mathrm{f}}(\xi)=\frac{\mathrm{V}_{1}\left(\xi_{\text {max }}-\xi_{\text {min }}\right)+\frac{\left(\mathrm{V}_{2}-\mathrm{V}_{1}\right)\left(\xi_{\max }^{\mathrm{p}+1}-\xi_{\text {min }}^{\mathrm{p}+1}\right)}{\mathrm{p}+1}}{\xi_{\text {max }}-\xi_{\text {min }}}
$$

the following equations can be obtained.

$$
\begin{aligned}
& \bar{V}_{f}(\xi)=V_{1}+\left(V_{2}-V_{1}\right) \frac{\left(\xi_{\max }^{p+1}-\xi_{\min }^{p+1}\right)}{\left(\xi_{\text {max }}-\xi_{\text {min }}\right)(p+1)} \\
& \bar{V}_{f}(\xi)=V_{1}+\left(V_{2}-V_{1}\right) Q \\
& \text { where } Q=\frac{\left(\xi_{\max }^{p+1}-\xi_{\min }^{p+1}\right)}{\left(\xi_{\max }-\xi_{\min }\right)(p+1)} \\
& \bar{V}_{f}(\xi)=V_{2} Q+V_{1}(1-Q) \\
& V_{2}=\frac{\bar{V}_{f}-V_{1}(1-Q)}{Q}
\end{aligned}
$$

The plots of the fibre ratio distribution for all ten optimisation cases considered in this paper were obtained using Eq. (11). It should be noted that each of the ten optimisation cases has a mean fibre ratio value of 0.4 . The case with power index of zero (i.e. $\mathrm{P}=0$ ) represents the traditional composite plate. This case has been included in all plots to show the differences between the 
fibre distribution in the FGC plate and the traditional composite plate. The case $\mathrm{P}=1$ and case $\mathrm{P}=2$ can be described as the linear fibre distribution case and the quadratic fibre distribution case respectively. The parameter $\mathrm{V}_{1}$ is use to offset the fibre ratio at edge $\mathrm{x}=0$ of the FGC plate.

The smooth fibre distribution plots for the ten optimisation cases are as shown in Figures 2 to 4 . These figures show the plot of fibre ratio at the Gaussian quadrature points along the $\mathrm{x}$-direction of the FGC plate.

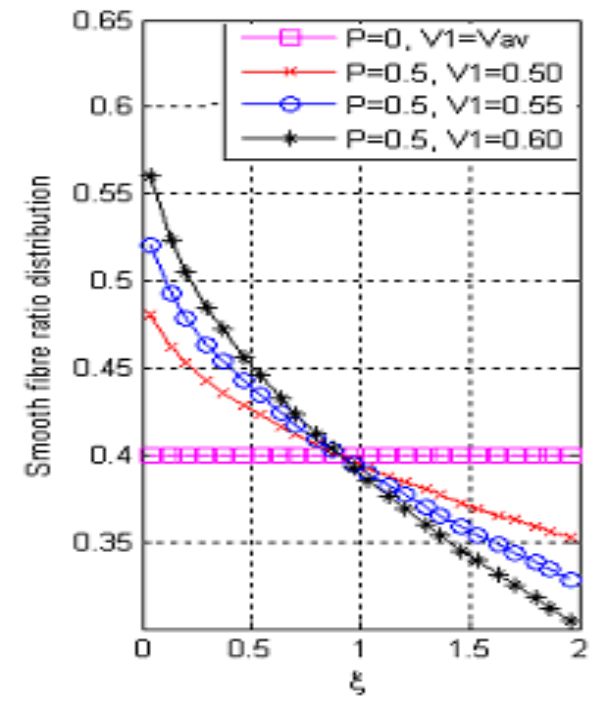

Figure 2: Smooth fibre ratio distribution plot for cases with $P=0$ and $P=0.5$

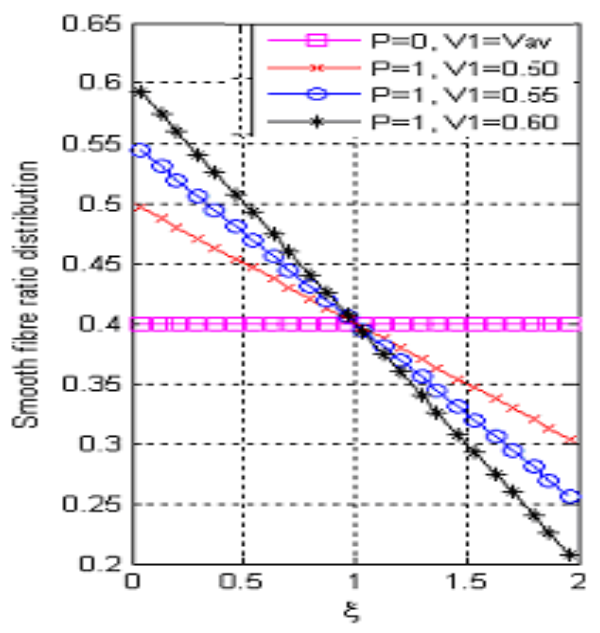

Figure 3: Smooth fibre ratio distribution plot for cases with $P=0$ and $P=1$

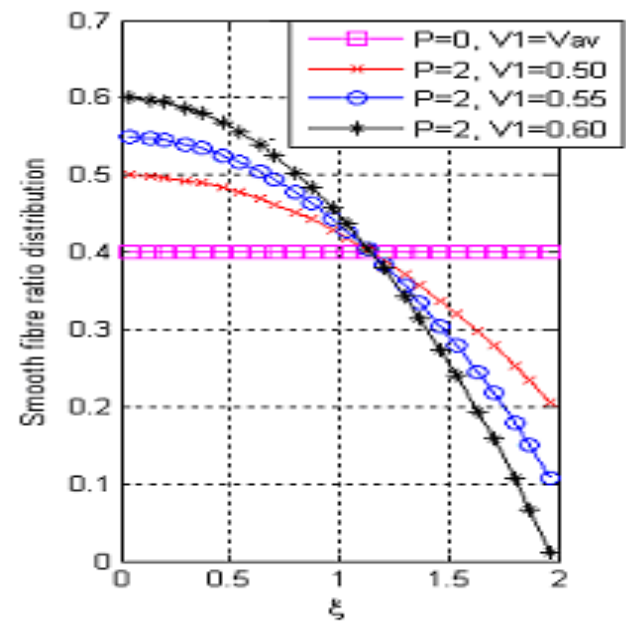

Figure 4: Smooth fibre ratio distribution plot for cases with $P=0$ and $P=2$

The average fibre distribution plots for the ten optimisation cases are as shown in Figures 5 to 7 . These figures show the plot of fibre ratios at the Gaussian quadrature points along the x-direction of the FGC plate. 


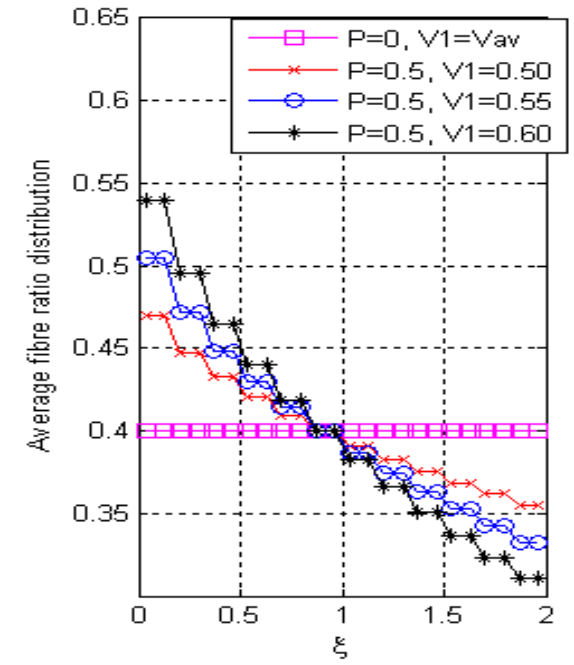

Figure 5: Average fibre ratio distribution plot for cases with $P=0$ and $P=0.5$

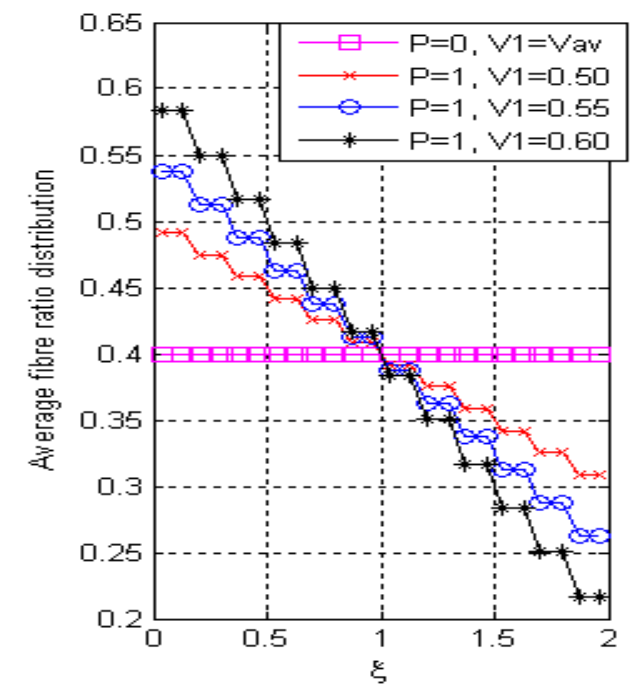

Figure 6: Average fibre ratio distribution plot for cases with $P=0$ and $P=1$

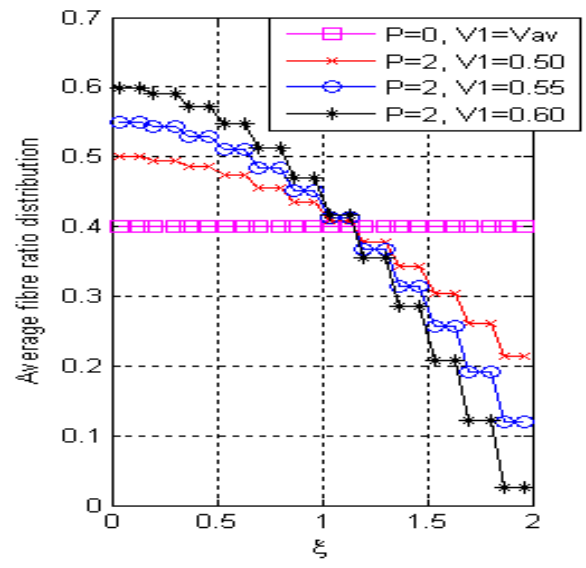

Figure 7: Average fibre ratio distribution plot for cases with $P=0$ and $P=2$

\section{Finite Element Equations for Mindlin-type}

\section{Element}

This section explains the stiffness matrix and mass matrix formulation for the Mindlin-type plate bending element theory.

\subsection{Finite Element Theory}

The authors [1] have previously written a paper on structural integrity of functionally graded composite structure using Mindlin-type finite elements. Further details of the Mindlin-type element theory can be found in the paper [1]. The theory includes the formulation of the displacement equation, strain equation, stress equation, strain energy variation and generalised equation of equilibrium. The generalised equation of equilibrium is then linearised, in-order to obtain the Mindin-type element equation. 


\subsection{Element Mass Matrix Derivation}

It is assumed that different layers are made of the same composite material but with different fibre orientations, i.e. the density of every layer is the same i.e.

$$
\rho^{(L)}=\rho
$$

Using D'Alembert's principle, the inertial force vector acting at an infinitesimal volume due to an acceleration vector is:

$$
d \vec{F}=-\rho \vec{a} d x d y d z
$$

Hence, the work done by the inertia force due to an infinitesimal virtual displacement field is:

$$
\begin{aligned}
d W_{a} & =-\iiint_{\text {volume }} \rho d \vec{q} \cdot \vec{a} d x d y d z \\
& \equiv-\iiint_{\text {volume }} \rho d \mathbf{q}^{t} \ddot{\mathbf{q}} d x d y d z
\end{aligned}
$$

Using the expression below

$$
\mathbf{q}(\mathrm{x}, \mathrm{y}, \mathrm{z}, \mathrm{t})=\left[\begin{array}{l}
\mathbf{q}_{u v} \\
\mathbf{q}_{w}
\end{array}\right]=\left[\begin{array}{c}
\mathbf{q}_{o}-z \mathbf{q}_{\theta} \\
\mathbf{q}_{w}
\end{array}\right]
$$

It can be shown that

$$
d \mathbf{q}^{t} \ddot{\mathbf{q}}=\left(d \mathbf{q}_{\mathbf{o}}^{t}-z d \mathbf{q}_{\theta}^{t}\right) *\left(\ddot{\mathbf{q}}_{\mathbf{o}}-z \ddot{\mathbf{q}}_{\theta}\right)+d \mathbf{q}_{\mathbf{w}}^{t} \ddot{\mathbf{q}}_{\mathbf{w}}
$$

Expanding the equation above and integrating with respect to $\mathrm{z}$ gives:

$$
\begin{gathered}
\int_{-h / 2}^{h / 2} d \mathbf{q}^{t} \ddot{\mathbf{q}} d z=h\left(d \mathbf{q}_{\mathbf{o}}^{t} \ddot{\mathbf{q}}_{\mathbf{o}}+d \mathbf{q}_{\mathbf{w}}^{t} \ddot{\mathbf{q}}_{\mathbf{w}}\right) \\
+\frac{h^{3}}{12} d \mathbf{q}_{\theta}^{t} \ddot{\mathbf{q}}_{\theta}
\end{gathered}
$$

Defining the following displacement components:

$$
\mathbf{q}_{o}(x, y, t)=\mathbf{N}_{o}(x, y) \boldsymbol{\delta}_{o}(t)
$$

$$
\begin{gathered}
\mathbf{q}_{\theta}(x, y, t)=\mathbf{N}_{\theta}(x, y) \boldsymbol{\delta}_{b}(t) \\
\mathbf{q}_{w}(x, y, t)=\mathbf{N}_{w}(x, y) \boldsymbol{\delta}_{b}(t) \\
\mathbf{N}_{o}(x, y) \equiv \mathbf{N}_{o}(\xi, \eta)=\left[\begin{array}{cccc}
\ldots & \mathbf{N}_{i}(\xi, \eta) & 0 & \ldots \\
\ldots & 0 & \mathrm{~N}_{i}(\xi, \eta) & \ldots
\end{array}\right] \\
\mathbf{N}_{\theta}(x, y) \equiv \mathbf{N}_{\theta}(\xi, \eta)=\left[\begin{array}{llccc}
\ldots & 0 & 0 & -\mathrm{N}_{i}(\xi, \eta) & \ldots \\
\ldots & 0 & \mathrm{~N}_{i}(\xi, \eta) & 0 & \ldots
\end{array}\right] \\
\mathbf{N}_{w}(x, y) \equiv \mathbf{N}_{w}(\xi, \eta)=\left[\begin{array}{lllll}
\ldots & \mathbf{N}_{i}(\xi, \eta) & 0 & 0 & \ldots
\end{array}\right]
\end{gathered}
$$

Equation (18) can be rewritten as:

$$
d W_{a}=-\iint_{x-y}\left\{\begin{array}{l}
\rho h d \boldsymbol{\delta}_{o}^{t} \mathbf{N}_{o}^{t} \mathbf{N}_{o} \ddot{\boldsymbol{\delta}}_{o} \\
+\rho h d \mathbf{\delta}_{b}^{t} \mathbf{N}_{w}^{t} \mathbf{N}_{w} \ddot{\boldsymbol{\delta}}_{b} \\
+\frac{\rho h^{3}}{12} d \boldsymbol{\delta}_{b}^{t} \mathbf{N}_{\theta}^{t} \mathbf{N}_{\theta} \ddot{\boldsymbol{\delta}}_{b}
\end{array}\right\} d x d y
$$

Defining the following mass matrices:

$$
\begin{aligned}
& \mathbf{M}_{o o}=\iint_{x-y} \rho h \mathbf{N}_{o}^{t}(\xi, \eta) \mathbf{N}_{o}(\xi, \eta) d x d y \\
& \mathbf{M}_{w w}=\iint_{x-y} \rho h \mathbf{N}_{w}^{t}(\xi, \eta) \mathbf{N}_{w}(\xi, \eta) d x d y \\
& \mathbf{M}_{\theta \theta}=\iint_{x-y} \frac{\rho h^{3}}{12} \mathbf{N}_{\theta}^{t}(\xi, \eta) \mathbf{N}_{\theta}(\xi, \eta) d x d y
\end{aligned}
$$

Equation (28) can be rewritten as:

$$
\begin{aligned}
d W_{a} & =-\left\{d \delta_{o}^{t} \mathbf{M}_{o o} \ddot{\boldsymbol{\delta}}_{o}+d \boldsymbol{\delta}_{b}^{t}\left[\mathbf{M}_{w w}+\mathbf{M}_{\theta \theta}\right] \ddot{\boldsymbol{\delta}}_{b}\right\} \\
& \equiv-d \boldsymbol{\delta}^{t} \mathbf{M} \ddot{\boldsymbol{\delta}}
\end{aligned}
$$

Hence it can be shown that the element mass matrix is as given below:

$$
\mathbf{M}=\left[\begin{array}{cc}
\mathbf{M}_{o o} & 0 \\
0 & \mathbf{M}_{w w}+\mathbf{M}_{\theta \theta}
\end{array}\right]
$$




\section{Finite Element Equations for Reissner-type}

\section{Element}

This section explains the Reissner-type plate bending element theory and summarises the equations used in the finite element programming. This theory defines the displacement equation, strain equation, stress equation, strain energy variation and generalised equation of equilibrium. The generalised equation of equilibrium is then linearised in-order to obtain the Reissner-type element equation. Finally, the stiffness matrix and mass matrix were derived for the Reissner-type plate bending element.

\subsection{Finite Element Theory}

\section{Displacement Equations}

The displacement components at the midplane of an $n$ node element can be subdivided into the following categories:

(i) In-plane components

$$
\begin{aligned}
& u^{o}(x, y)=\sum_{i=1}^{n} N_{i}(x, y) u_{i}^{o} \\
& v^{o}(x, y)=\sum_{i=1}^{n} N_{i}(x, y) v_{i}^{o}
\end{aligned}
$$

where $N_{i}$ represents Lagrangian shape functions.

(ii) Transverse shear components

$$
\psi_{x}(x, y)=\sum_{i=1}^{n} N_{i}(x, y)\left(\psi_{x}\right)_{i}
$$

$$
\psi_{y}(x, y)=\sum_{i=1}^{n} N_{i}(x, y)\left(\psi_{y}\right)_{i}
$$

where $\psi_{x}$ and $\psi_{y}$ represents the average transverse shear deformation in the $\mathrm{x}-\mathrm{z}$ and $\mathrm{y}-\mathrm{z}$ plane respectively.

(iii) Out-of-plane component

There are two types of interpolation for the lateral deflection $w$ :

(a) Non-conforming elements

$$
w(x, y)=\sum_{i=1}^{n}\left[F_{i}(x, y) w_{i}+G_{i}(x, y) w_{i, x}+H_{i}(x, y) w_{i, y}\right]
$$

(b) Conforming elements

$$
w(x, y)=\sum_{i=1}^{n}\left[\begin{array}{l}
F_{i}(x, y) w_{i}+G_{i}(x, y) w_{i, x} \\
+H_{i}(x, y) w_{i, y}+P_{i}(x, y) w_{i, x y}
\end{array}\right]
$$

where $F_{i}, G_{i}, H_{i}, P_{i}$ represent Hermitian shape functions. The explicit expressions for the Lagrangian and Hermitian shape functions can be found in [13-14] $\underline{\text { Transverse shear strain components }}$

These strain components are assumed infinitesimal and are represented by the equation shown below.

$$
\hat{\boldsymbol{\gamma}}(x, y)=\left[\begin{array}{c}
-\psi_{y} \\
\psi_{x}
\end{array}\right] \equiv \mathbf{B}_{\gamma}(x, y) \boldsymbol{\delta}_{\psi}
$$

where

$$
\mathbf{B}_{\gamma}(x, y)=\left[\begin{array}{cccc}
\cdots & 0 & -N_{i}(x, y) & \cdots \\
\cdots & N_{i}(x, y) & 0 & \cdots
\end{array}\right]
$$

$$
\boldsymbol{\delta}_{\psi}=\left\{\left(\psi_{x}\right)_{1}\left(\psi_{y}\right)_{1}\left(\psi_{x}\right)_{2}\left(\psi_{y}\right)_{2} \cdots\left(\psi_{x}\right)_{n}\left(\psi_{y}\right)_{n}\right\}
$$

$\underline{\mathrm{x}-\mathrm{y} \text { strain components }}$ 
These strain components can be obtained from Green's strain-displacement equations i.e. Equations (43 - 45). They can be divided into two parts which include the infinitesimal component derived from the Cauchy's strain-displacement equation, and the additional nonlinear terms in Green's equation.

$$
\begin{aligned}
& \varepsilon_{x}=\frac{\partial u}{\partial x}+\frac{1}{2}\left[\left(\frac{\partial u}{\partial x}\right)^{2}+\left(\frac{\partial v}{\partial x}\right)^{2}+\left(\frac{\partial w}{\partial x}\right)^{2}\right] \\
& \varepsilon_{y}=\frac{\partial v}{\partial y}+\frac{1}{2}\left[\left(\frac{\partial u}{\partial y}\right)^{2}+\left(\frac{\partial v}{\partial y}\right)^{2}+\left(\frac{\partial w}{\partial y}\right)^{2}\right] \\
& \gamma_{x y}=\frac{\partial u}{\partial y}+\frac{\partial v}{\partial x}+\left[\frac{\partial u}{\partial x} \frac{\partial u}{\partial y}+\frac{\partial v}{\partial x} \frac{\partial v}{\partial y}+\frac{\partial w}{\partial x} \frac{\partial w}{\partial y}\right]
\end{aligned}
$$

The total strain vector can be obtained in terms of the nodal parameters and shape function by substituting the above displacement and slope components into the Green's strain-displacement equations. The total strain vector equation above can then be manipulated to obtain the variation of strain vector in terms of the nodal parameters and shape functions.

\section{$\underline{\text { Strain Energy Variation }}$}

The variation of strain energy density at a point inside the $\mathrm{L}^{\text {th }}$ layer is given below.

$$
d \overline{\mathbf{U}}^{(L)}=d \boldsymbol{\gamma}^{t} \boldsymbol{\tau}^{(L)}+d \boldsymbol{\varepsilon}^{t} \boldsymbol{\sigma}^{(L)}
$$

\section{$\underline{\text { Generalised Equation of Equilibrium }}$}

The work done by actual loads can be expressed in terms of equivalent nodal loads as given below.

$$
d W=d \boldsymbol{\delta}_{o}^{t} \mathbf{F}_{o}+d \boldsymbol{\delta}_{b}^{t} \mathbf{F}_{b}
$$

Using the principle of virtual work, the generalised equation of equilibrium can be derived.

$$
d U-d W=0
$$

An approximate solution of this equation of equilibrium gives the expression for the residual vector.

\section{$\underline{\text { Linearisation of Equations of Equilibrium and Derivation }}$} of Element Equations

In order to restore equilibrium, the residual vector must approach a value of zero. This equilibrium is achieved by employing the expressions below.

$$
\begin{aligned}
& \boldsymbol{\delta}_{\text {new }}=\boldsymbol{\delta}_{\text {old }}+\Delta \boldsymbol{\delta} \\
& \boldsymbol{\sigma}_{\text {new }}^{(L)}=\boldsymbol{\sigma}_{\text {old }}+\Delta \boldsymbol{\sigma} \\
& \mathbf{A}_{\text {new }}=\mathbf{A}_{\text {old }}+\Delta \mathbf{A}
\end{aligned}
$$

The combination of the above expressions and the residual vector expressions results in a final matrix equation, which is given below.

$$
\sum_{e=1}^{N_{e}}\left\{\left(\mathbf{K}+\mathbf{K}^{\sigma}\right)\left[\begin{array}{c}
\Delta \boldsymbol{\delta}_{o} \\
\Delta \boldsymbol{\delta}_{b}
\end{array}\right]\right\}=\left[\begin{array}{l}
\mathbf{R}_{o} \\
\mathbf{R}_{b}
\end{array}\right]
$$

\subsection{Element Mass Matrix Derivation}

This derivation follows the same procedure as in section 3.2. Hence Eqs.16-18 also applies to this section. Using the displacement expression below

$\mathbf{q}(x, y, z, t)=\left[\begin{array}{l}\mathbf{q}_{u v} \\ \mathbf{q}_{w}\end{array}\right]=\left[\begin{array}{c}\mathbf{q}_{o}-z \mathbf{q}_{\theta}-f_{\psi} \mathbf{q}_{\psi} \\ \mathbf{q}_{w}\end{array}\right]$

It can be shown that 


$$
\begin{aligned}
d \mathbf{q}^{t} \ddot{\mathbf{q}} & =\left(d \mathbf{q}_{\mathbf{o}}^{t}-z d \mathbf{q}_{\theta}^{t}-f_{\psi} d \mathbf{q}_{\psi}^{t}\right) \\
& *\left(\ddot{\mathbf{q}}_{\mathbf{o}}-z \ddot{\mathbf{q}}_{\theta}-f_{\psi} \ddot{\mathbf{q}}_{\psi}\right)+d \mathbf{q}_{\mathbf{w}}^{t} \ddot{\mathbf{q}}_{\mathbf{w}}
\end{aligned}
$$

Expanding the equation above and integrating with respect to $\mathrm{z}$ gives:

$$
\begin{aligned}
\int_{-h / 2}^{h / 2} d \mathbf{q}^{t} \ddot{\mathbf{q}} d z= & h\left(d \mathbf{q}_{\mathbf{o}}^{t} \ddot{\mathbf{q}_{\mathbf{o}}}+d \mathbf{q}_{\mathbf{w}}^{t} \ddot{\mathbf{q}}_{\mathbf{w}}\right) \\
& +\frac{h^{3}}{12} d \mathbf{q}_{\theta}^{t} \ddot{\mathbf{q}}_{\theta}+\frac{17 h^{3}}{140} d \mathbf{q}_{\psi}^{t} \ddot{\mathbf{q}}_{\psi} \\
& +\frac{h^{3}}{10}\left(d \mathbf{q}_{\theta}^{t} \ddot{\mathbf{q}}_{\psi}+d \mathbf{q}_{\psi}^{t} \ddot{\mathbf{q}}_{\theta}\right)
\end{aligned}
$$

Defining the following displacement components:

$$
\begin{aligned}
& \mathbf{q}_{o}(x, y, t)=\mathbf{N}_{o}(x, y) \boldsymbol{\delta}_{o}(t) \\
& \mathbf{q}_{\theta}(x, y, t)=\mathbf{N}_{\theta}(x, y) \boldsymbol{\delta}_{b}(t) \\
& \mathbf{q}_{\psi}(x, y, t)=\mathbf{N}_{\psi}(x, y) \boldsymbol{\delta}_{t}(t) \\
& \mathbf{q}_{w}(x, y, t)=\mathbf{N}_{w}(x, y) \boldsymbol{\delta}_{b}(t) \\
& \mathbf{N}_{o}(x, y) \equiv \mathbf{N}_{o}(\xi, \eta)=\left[\begin{array}{cccc}
\ldots & \mathbf{N}_{i}(\xi, \eta) & 0 & \ldots \\
\ldots & 0 & \mathbf{N}_{i}(\xi, \eta) & \ldots
\end{array}\right] \\
& \mathbf{N}_{\psi}(x, y) \equiv \mathbf{N}_{\psi}(\xi, \eta)=\left[\begin{array}{cccc}
\ldots & 0 & \mathbf{N}_{i}(\xi, \eta) & \ldots \\
\ldots & -\mathbf{N}_{i}(\xi, \eta) & 0 & \ldots
\end{array}\right] \\
& \mathbf{N}_{w}(x, y) \equiv \mathbf{N}_{w}(\xi, \eta)=\left[\begin{array}{llllll}
\ldots & F_{i} & H_{i} & -G_{i} & P_{i} & \ldots
\end{array}\right] \\
& \mathbf{N}_{\theta}(x, y)=\left[\begin{array}{l}
\frac{\partial v}{\partial x} \\
\frac{\partial w}{\partial y}
\end{array}\right]=\left[\begin{array}{llllll}
\ldots & F_{i, x} & H_{i, x} & -G_{i, x} & P_{i, x} & \ldots \\
\ldots & F_{i, y} & H_{i, y} & -G_{i, y} & P_{i, y} & \ldots
\end{array}\right]
\end{aligned}
$$

Equation (18) can be rewritten as:

$$
d W_{a}=-\iint_{x-y}\left\{\begin{array}{l}
\rho h d \mathbf{\delta}_{o}^{t} \mathbf{N}_{o}^{t} \mathbf{N}_{o} \ddot{\boldsymbol{\delta}}_{o}+\rho h d \mathbf{\delta}_{b}^{t} \mathbf{N}_{w}^{t} \mathbf{N}_{w} \ddot{\boldsymbol{\delta}}_{b} \\
+\frac{\rho h^{3}}{12} d \boldsymbol{\delta}_{b}^{t} \mathbf{N}_{\theta}^{t} \mathbf{N}_{\theta} \ddot{\boldsymbol{\delta}}_{b} \\
+\frac{17 \rho h^{3}}{140} d \delta_{t}^{t} \mathbf{N}_{\psi}^{t} \mathbf{N}_{\psi} \ddot{\boldsymbol{\delta}}_{t} \\
+\frac{\rho h^{3}}{10}\left(d d \mathbf{\delta}_{b}^{t} \mathbf{N}_{\theta}^{t} \mathbf{N}_{\psi} \ddot{\boldsymbol{\delta}}_{t}+d \mathbf{\delta}_{t}^{t} \mathbf{N}_{\psi}^{t} \mathbf{N}_{\theta} \ddot{\boldsymbol{\delta}}_{b}\right)
\end{array}\right\} d x d y
$$

Defining the following mass matrices:

$$
\begin{aligned}
& \mathbf{M}_{o o}=\iint_{x-y} \rho h \mathbf{N}_{o}^{t}(\xi, \eta) \mathbf{N}_{o}(\xi, \eta) d x d y \\
& \mathbf{M}_{w w}=\iint_{x-y} \rho h \mathbf{N}_{w}^{t}(\xi, \eta) \mathbf{N}_{w}(\xi, \eta) d x d y
\end{aligned}
$$

$\mathbf{M}_{\theta \theta}=\iint_{x-y} \frac{\rho h^{3}}{12} \mathbf{N}_{\theta}^{t}(\xi, \eta) \mathbf{N}_{\theta}(\xi, \eta) d x d y$

$$
\mathbf{M}_{\psi \psi}=\iint_{x-y} \frac{17 \rho h^{3}}{140} \mathbf{N}_{\psi}^{t}(\xi, \eta) \mathbf{N}_{\psi}(\xi, \eta) d x d y
$$

$$
\mathbf{M}_{\psi \theta}=\iint_{x-y} \frac{\rho h^{3}}{10} \mathbf{N}_{\psi}^{t}(\xi, \eta) \mathbf{N}_{\theta}(\xi, \eta) d x d y
$$

$$
\mathbf{M}_{\theta \psi}=\iint_{x-y} \frac{\rho h^{3}}{10} \mathbf{N}_{\theta}^{t}(\xi, \eta) \mathbf{N}_{\psi}(\xi, \eta) d x d y \equiv \mathbf{M}_{\psi \theta}^{t}
$$

Equation (62) can be rewritten as:

$$
\begin{aligned}
& d W_{a}=-\left\{\begin{array}{l}
d \boldsymbol{\delta}_{o}^{t} \mathbf{M}_{o o} \ddot{\boldsymbol{\delta}}_{o}+d \boldsymbol{\delta}_{b}^{t}\left[\mathbf{M}_{w w}+\mathbf{M}_{\theta \theta}\right] \ddot{\boldsymbol{\delta}}_{b} \\
+d \boldsymbol{\delta}_{t}^{t} \mathbf{M}_{\psi \psi} \ddot{\boldsymbol{\delta}}_{t} \\
+\left(d \boldsymbol{\delta}_{b}^{t} \mathbf{M}_{\theta \psi} \ddot{\boldsymbol{\delta}}_{t}+d \delta_{t}^{t} \mathbf{M}_{\psi \theta} \ddot{\boldsymbol{\delta}}_{b}\right)
\end{array}\right\} \\
& \equiv-d \boldsymbol{\delta}^{t} \mathbf{M} \ddot{\boldsymbol{\delta}}
\end{aligned}
$$

Hence it can be shown that the element mass matrix is as given below: 


$$
\mathbf{M}=\left[\begin{array}{ccc}
\mathbf{M}_{o o} & \mathbf{0} & \mathbf{0} \\
\mathbf{0} & \mathbf{M}_{w w}+\mathbf{M}_{\theta \theta} & \mathbf{M}_{\theta \psi} \\
\mathbf{0} & \mathbf{M}_{\psi \theta} & \mathbf{M}_{\psi \psi}
\end{array}\right]
$$

\section{Buckling Analysis: Procedure and}

\section{Numerical Example}

\subsection{Buckling Analysis Solution}

Buckling occurs at infinitesimal strains.

$$
\begin{aligned}
& \boldsymbol{\sigma}_{\mathrm{L}} \Rightarrow \mathbf{0} \\
& \mathbf{F}_{\mathrm{L}} \Rightarrow \mathbf{0} \\
& \boldsymbol{\sigma}_{\mathrm{m}}=\mathbf{D}_{\mathrm{oo}} \boldsymbol{\varepsilon}_{\mathrm{o}}-\mathbf{D}_{\mathrm{ob}} \boldsymbol{\varepsilon}_{\mathrm{b}} \\
& \boldsymbol{\sigma}_{\theta}=\mathbf{D}_{\mathrm{bb}} \boldsymbol{\varepsilon}_{\mathrm{o}}-\mathbf{D}_{(3)} \boldsymbol{\varepsilon}_{\mathrm{b}} \\
& \boldsymbol{\sigma}_{\mathrm{m} \theta}=-\mathbf{D}_{\mathrm{ob}} \boldsymbol{\varepsilon}_{\mathrm{o}}-\mathbf{D}_{\mathrm{bb}} \boldsymbol{\varepsilon}_{\mathrm{b}}
\end{aligned}
$$

A small deflection analysis can be carried out with a small load representing the distribution of actual load, and has equivalent nodal loading vector $\mathbf{F}_{\mathrm{o}}$ which is defined below.

$$
\mathbf{F}_{\mathrm{o}}=\mathbf{K} \boldsymbol{\delta}_{\mathrm{o}}
$$

Just before the onset of instability, the strains can be considered infinitesimal, and if instability occurs at:

$$
\mathbf{F}=\lambda \mathbf{F}_{\mathrm{o}}
$$

Hence

$$
\begin{gathered}
\left\{\mathbf{K}+\lambda \mathbf{K}^{\sigma}\left(\sigma_{\mathrm{o}}\right)\right\} \boldsymbol{\delta} \Rightarrow \mathbf{0} \\
\left|\mathbf{K}+\lambda \mathbf{K}^{\sigma}\left(\sigma_{\mathrm{o}}\right)\right|=0
\end{gathered}
$$

This gives a characteristic equation and its smallest real roots define the critical buckling load.

$$
\mathbf{F}_{\text {critical }}=\lambda_{\text {min }} \mathbf{F}_{\mathrm{o}}
$$

\subsection{Numerical Example of Buckling Case}

\section{Study}

\section{Composite Material Data}

The composite material data used for all the cases studies are tabulated below.

Table 1: Composite material data

\begin{tabular}{|l|l|}
\hline Parameters & Values \\
\hline $\mathrm{E}_{\mathrm{f}}$, Fibre young modulus & $330 \mathrm{GPa}$ \\
\hline $\mathrm{E}_{\mathrm{m}}$, Matrix young modulus & $5 \mathrm{GPa}$ \\
\hline$v_{\mathrm{f}}$, Fibre Poisson ratio & 0.3625 \\
\hline$v_{\mathrm{m}}$, Matrix Poisson ratio & 0.3 \\
\hline$\rho_{\mathrm{f}}$, Fibre density & $1.0 \mathrm{Kg} / \mathrm{m}^{3}$ \\
\hline$\rho_{\mathrm{m}}$, Matrix density & $1.0 \mathrm{Kg} / \mathrm{m}^{3}$ \\
\hline Stacking sequence & $\left((-45,0,45)_{2}\right)_{\mathrm{S}}$ \\
\hline Ply thickness & $2.5 \mathrm{~mm}$ \\
\hline $\mathrm{X}_{\mathrm{MT}}$, Matrix tensile strength & $0.05 \mathrm{MPa}$ \\
\hline $\mathrm{X}_{\mathrm{MC}}$, Matrix compressive strength & $0.08 \mathrm{MPa}$ \\
\hline $\mathrm{X}_{\mathrm{MS}}$, Matrix shear strength & $0.04 \mathrm{MPa}$ \\
\hline $\mathrm{X}_{\mathrm{FT}}$, Fibre tensile strength & $7.5 \mathrm{MPa}$ \\
\hline $\mathrm{X}_{\mathrm{FS}}$, Fibre shear strength & $4 \mathrm{MPa}$ \\
\hline $\mathrm{S}_{\mathrm{EGRM}}$, Maximum radial residual & 0.0 \\
\hline $\mathrm{S}_{\mathrm{KC}}$, Longitudinal stress & \\
\hline
\end{tabular}




\begin{tabular}{|l|l|}
\hline$\overline{\mathrm{V}}$, Average fibre ratio & 0.4 \\
\hline $\mathrm{P}$, Power term in the fibre & $0,0.5,1$ or 2 \\
distribution equation & \\
\hline
\end{tabular}

A rectangular plate made of a typical FGM with its mid-plane as shown in Figure 8 was considered. The width-to-thickness ratio of the plate is 33 . A 72 element mesh was employed for all the case studies. The elements used in this exercise include Mindlin-type element and Reissner-type element. The boundary condition applied in the case studies is that edge $\mathrm{x}=0$ is a clamped edge. A load of $0.1 \mathrm{kN}$ was applied as an equivalent nodal loading at edge $\mathrm{x}=2$ for the buckling case study.

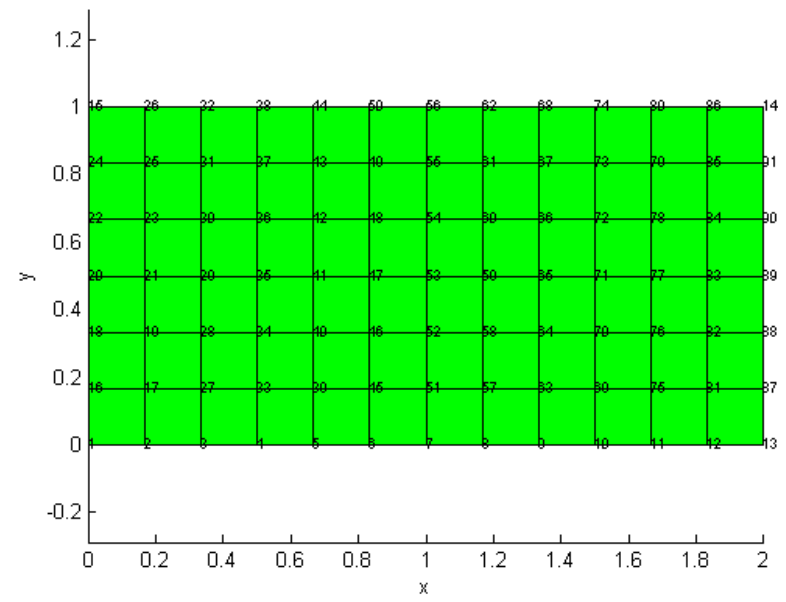

Figure 8: Mesh

Table 2, shows the comparison of the buckling eigenvalue results for all ten fibre ratio distribution cases. Maximisation of the buckling eigenvalue for most buckling modes is usually the desired effect required for design purposes. On looking at the table below, it can be seen that the first and second buckling mode results of the Reissner and Mindlin programs are in good agreement. But the third buckling mode results of the Reissner and Mindlin programs are not in good agreement. This discrepancy can be attributed to the difference transverse modelling technique employed by both elements. Also the discrepancy could be reduced by using a finer mesh of the Mindlin-type element which results in the convergence of the Mindlin-type element solution to Reissner-type element solution. This is due to the fact that the Reissner-type element (based on parabolic transverse shear modelling through the plate thickness) gives a more accurate result in comparison with the Mindlin-type element (based on average transverse shear modelling through the plate thickness). In the high buckling mode cases (such as the $3^{\text {rd }}$ buckling mode) or the non-linear fibre distribution cases (i.e. $\mathrm{P}=0.5$ and $\mathrm{P}=2$ ), FE solution is mesh-sensitive due to the nonlinearity of the stiffness property distribution in the plate longitudinal direction. Hence convergence study must be carried out for non-linear fibre distribution cases and high buckling mode cases when using any of the elements. Finally, in this buckling analysis, an average desirable effect of $20 \%$ increment in critical buckling load (relative to the traditional composite case) for the first and second buckling mode is achieved by using functionally graded composite. 
Table 2: Buckling Eigenvalue

\begin{tabular}{|c|c|c|c|c|}
\hline \multicolumn{5}{|c|}{ Buckling Eigenvalue, $\lambda_{\min }=\mathrm{F}_{\text {critical }} / \mathrm{F}_{\mathrm{o}}$} \\
\hline $\mathrm{P}$ & V1 & $\begin{array}{l}1^{\text {st }} \text { Mode } \\
\text { (Mindlin) }\end{array}$ & $\begin{array}{l}2^{\text {nd }} \text { Mode } \\
\text { (Mindlin) }\end{array}$ & $\begin{array}{l}3^{\text {rd }} \text { Mode } \\
\text { (Mindlin) }\end{array}$ \\
\hline 0 & $\mathrm{Vav}=0.4$ & 8.130 & 75.021 & 145.28 \\
\hline 0.5 & 0.5 & 8.504 & 75.482 & 150.91 \\
\hline 0.5 & 0.55 & 8.665 & 75.452 & 184.14 \\
\hline 0.5 & 0.6 & 8.808 & 75.256 & 181.92 \\
\hline 1 & 0.5 & 8.752 & 75.045 & 183.72 \\
\hline 1 & 0.55 & 9.006 & 74.364 & 169.74 \\
\hline 1 & 0.6 & 9.221 & 73.170 & 153.49 \\
\hline 2 & 0.5 & 9.072 & 73.919 & 161.63 \\
\hline 2 & 0.55 & 9.439 & 71.192 & 130.65 \\
\hline 2 & 0.6 & 9.690 & 65.436 & 93.401 \\
\hline $\mathrm{P}$ & V1 & $\begin{array}{l}1^{\text {st }} \text { Mode } \\
\text { (Reissner) }\end{array}$ & $\begin{array}{l}2^{\text {nd }} \text { Mode } \\
\text { (Reissner) }\end{array}$ & $\begin{array}{l}3^{\text {rd }} \text { Mode } \\
\text { (Reissner) }\end{array}$ \\
\hline 0 & $\mathrm{Vav}=0.4$ & 8.135 & 73.154 & 204.33 \\
\hline 0.5 & 0.5 & 8.513 & 73.643 & 196.38 \\
\hline 0.5 & 0.55 & 8.675 & 73.622 & 189.50 \\
\hline 0.5 & 0.6 & 8.820 & 73.433 & 181.90 \\
\hline 1 & 0.5 & 8.766 & 73.231 & 184.33 \\
\hline 1 & 0.55 & 9.024 & 72.586 & 169.26 \\
\hline 1 & 0.6 & 9.244 & 71.441 & 152.93 \\
\hline 2 & 0.5 & 9.094 & 72.246 & 161.14 \\
\hline 2 & 0.55 & 9.477 & 69.808 & 129.62 \\
\hline 2 & 0.6 & 9.767 & 65.177 & 94.007 \\
\hline
\end{tabular}

\section{Dynamic Analysis: Procedure and}

\section{Numerical Example}

\subsection{Dynamic Analysis Solution}

Consider an ideal structure with no damping forces, the dynamic finite element matrix equation can be reduced to the expression below.

$$
\mathbf{M} \ddot{\boldsymbol{\delta}}(\mathrm{t})+\mathbf{K} \boldsymbol{\delta}(\mathrm{t})=\mathbf{F}(\mathrm{t})
$$

In a natural mode, each point of a structure executes harmonic motion about the position of static equilibrium at the same frequency. Hence it can be assumed that, at a natural mode of vibration:

$$
\boldsymbol{\delta}(\mathrm{t})=\tilde{\boldsymbol{\delta}} \cos (\omega \mathrm{t})
$$

where $\underline{\tilde{\delta}}$ represents the vector of nodal amplitudes.

The matrix equation represents a system of homogenous simultaneous equations which can have a non-trivial solution, if the value of $\lambda$ satisfies the condition below:

$$
|\mathbf{K}-\lambda \mathbf{M}|=0
$$

where $\lambda=\omega^{2}$

On comparing natural frequency problem and buckling problem, it can be shown that both problems are equivalent and they are related to each other through the expression below.

$$
\mathbf{M}^{\prime}=-\mathbf{K}^{\sigma}\left(\sigma_{\mathrm{o}}\right)
$$

The matrix equation of the dynamic eigenvalue problem was solved in this report. This report employed two 
techniques which include the simple iteration and subspace iteration techniques [15 - 17].

\subsection{Numerical Example of Vibration Case}

\section{Study}

The FE model used in this section is as described in Section 5.2. Table 3, shows the comparison of the natural vibration frequency results for all ten fibre ratio distribution cases. Maximisation of the natural frequency for most free vibration modes is usually the desired effect required for design purposes. On looking at the table below, it can be seen that the results of the Reissner and Mindlin programs are in good agreement for all vibration modes. Also the natural frequency increases as the exponent $\mathrm{P}$ values increases. This is expected because the stiffness at the root region of the composite plate increases with increase in exponent $\mathrm{P}$ value. Finally, in this vibration analysis, an average desirable effect of $15 \%$ increment in natural frequency (relative to the traditional composite case) for all the vibration mode is achieved by using this functionally graded composite.

Table 3: Natural vibration frequencies

\begin{tabular}{|l|l|l|l|l|}
\hline \multicolumn{5}{|c|}{ Natural Vibration Frequencies (Hz) } \\
\hline P & V1 & $1^{\text {st }}$ Mode & $2^{\text {nd }}$ Mode & $3^{\text {rd }}$ Mode \\
& & (Mindlin) & (Mindlin) & (Mindlin) \\
\hline 0 & Vav=0.4 & 299.90 & 1252.1 & 1862.8 \\
\hline 0.5 & 0.5 & 312.37 & 1269.2 & 1884.8 \\
\hline
\end{tabular}

\begin{tabular}{|c|c|c|c|c|}
\hline 0.5 & 0.55 & 318.12 & 1276.0 & 1893.4 \\
\hline 0.5 & 0.6 & 323.60 & 1281.7 & 1900.4 \\
\hline 1 & 0.5 & 319.13 & 1282.0 & 1894.7 \\
\hline 1 & 0.55 & 327.94 & 1292.7 & 1905.2 \\
\hline 1 & 0.6 & 336.31 & 1300.4 & 1912.0 \\
\hline 2 & 0.5 & 325.83 & 1300.4 & 1913.3 \\
\hline 2 & 0.55 & 337.79 & 1314.4 & 1927.2 \\
\hline 2 & 0.6 & 349.19 & 1317.0 & 1930.6 \\
\hline $\bar{P}$ & V1 & $\begin{array}{l}1^{\text {st }} \text { Mode } \\
\text { (Reissner) }\end{array}$ & $\begin{array}{l}2^{\text {nd }} \text { Mode } \\
\text { (Reissner) }\end{array}$ & $\begin{array}{l}3^{\text {rd }} \text { Mode } \\
\text { (Reissner) }\end{array}$ \\
\hline 0 & $\mathrm{Vav}=0.4$ & 300.24 & 1257.3 & 1832.3 \\
\hline 0.5 & 0.5 & 312.86 & 1275.2 & 1855.8 \\
\hline 0.5 & 0.55 & 318.67 & 1282.2 & 1864.9 \\
\hline 0.5 & 0.6 & $\begin{array}{l}\text { No } \\
\text { solution }\end{array}$ & $\begin{array}{l}\text { No } \\
\text { solution }\end{array}$ & $\begin{array}{l}\text { No } \\
\text { solution }\end{array}$ \\
\hline 1 & 0.5 & 319.64 & 1287.9 & 1865.7 \\
\hline 1 & 0.55 & 328.53 & 1298.7 & 1876.9 \\
\hline 1 & 0.6 & 336.96 & 1306.4 & 1884.6 \\
\hline 2 & 0.5 & 326.34 & 1305.9 & 1884.8 \\
\hline 2 & 0.55 & 338.40 & 1319.6 & 1900.2 \\
\hline 2 & 0.6 & $\begin{array}{l}\text { No } \\
\text { solution }\end{array}$ & $\begin{array}{l}\text { No } \\
\text { solution }\end{array}$ & $\begin{array}{l}\text { No } \\
\text { solution }\end{array}$ \\
\hline
\end{tabular}

\section{CONCLUSION}

In this paper, the optimum design criterion employed, is one that satisfies the follow design criteria. 
- Maximum critical buckling load (i.e. maximum buckling eigenvalue)

- Maximum vibration frequency.

Hence, using the definition of design criterion above, the results in the previous sections have been summarised below and the optimum design was determined from the table.

Table 4: Optimum Design

\begin{tabular}{|c|c|c|c|c|}
\hline & Elements & $\mathrm{P}$ & V1 & $\begin{array}{l}1^{\text {st }} \text { Mode } \\
\text { Results }\end{array}$ \\
\hline Buckling & Mindlin & 2 & 0.6 & $\begin{array}{l}\lambda_{\text {fgm }} / \lambda_{\text {comp }} \\
=1.19\end{array}$ \\
\hline Buckling & Reissner & 2 & 0.6 & $\begin{array}{l}\lambda_{\text {fgm }} / \lambda_{\text {comp }} \\
=1.2\end{array}$ \\
\hline $\begin{array}{l}\text { Free } \\
\text { vibration }\end{array}$ & Mindlin & 2 & 0.6 & $\begin{array}{l}\omega_{\text {fgm }} / \omega_{\text {comp }} \\
=1.17\end{array}$ \\
\hline $\begin{array}{l}\text { Free } \\
\text { vibration }\end{array}$ & Reissner & 2 & 0.55 & $\begin{array}{l}\omega_{\text {fgm }} / \omega_{\text {comp }} \\
=1.13\end{array}$ \\
\hline
\end{tabular}

Table 4 shows the optimum fibre distribution for each load case. Analysis of the results shows that fibre distribution with $\mathrm{P}=2$ and $\mathrm{V} 1=0.6$ is the optimum design. This fibre distribution has resulted in $20 \%$ increase in critical buckling load and $15 \%$ increase in the natural frequency of the composite structure.

In this paper, a Reissner-type element and a Mindlintype element have been formulated and used in performing a buckling and vibration analysis of a functionally graded composite structure. Some of the unique contributions achieved in this paper include Mindlin-type element formulation, Reissner-type element formulation, finite strain modelling and smooth fibre distribution technique. A methodical approach was used in demonstrating the design optimisation process. This involves undertaken vibration and buckling analysis for different fibre distribution cases and the effects of fibre distribution were studied. Fibre distribution cases with maximum vibration frequency and maximum buckling loads were chosen as the optimum design. Also this paper achieved its objective by presenting a detailed explanation of the functional graded technology from theoretical concept through to optimum design application. Future work recommendation would be to extend this work to cover non-linear dynamics and thermo-elasticity.

\section{REFERENCES}

[1] Oyekoya O.O., Mba D.U. and El-Zafrany A.M., 2008; Structural integrity of functionally graded composite structure using Mindlin-type finite elements. ICCES, vol.172, no.1, pp. 1-6.

[2] Chen X. L. and Liew K. M., 2004; Buckling of rectangular functionally graded material plates subjected to nonlinearly distributed in-plane edge loads. Smart Materials and Structures. 
[3] Reddy J. N., 2000; Analysis of functionally graded plates. International Journal of Numerical Methods in Engineering; 47: 663-684

[4] Javaheri R. and Eslami M.R. 2002; Buckling of functionally graded plates under in-plane compressive loading. Z. Angew. Math. Mech. 82, 4, $277-283$

[5] Shi J. and Askew C., 1997; Thermal stress in cylinders made of composite materials. Key Engineering Materials, Vols 127-131, pp. 1109-1116

[6] Silvia E.C.N. and Paulino G.H., 2004; Topology optimisation applied to the design of functionally graded material (FGM) structures; XXI ICTAM, Warsaw, Poland.

[7] Durodola J.F. and Adlington J.E., 1997; Functionally graded material properties for disks and rotors; Key Engineering Materials Vols. 127-131, pp. 1199-1206.

[8] Nogata F., 1995; Intelligent functionally graded material: Bamboo. Composites Engineering Vol. 5, No 7, pp. 743-751.

[9] Pompe W., Worch H., Epple M., Friess W., Gelinsky M., Greil P., Hempel U., Scharnweber D. and Schulte K., 2003; Functionally graded materials for biomedical applications. Materials Science and Engineering, A362, 40-60.

[10] Paulino G.H. and Kim J., 2004; A new approach to compute T-stress in functionally graded materials by means of the interaction integral method. Engineering Fracture Mechanics, 71, 1907-1950.

[11] Yang Y.Y., 1998; Stress analysis in a joint with a functionally graded material under a thermal loading by using the Mellin transform method. Int. J. Solids Structures, Vol. 35, No. 12 pp 1261-1287.

[12] Agarwal B.D. and Broutman L.J., 1990; Analysis and performance of fiber composites. $2^{\text {nd }}$ edition.

[13]El-Zafrany A. and Cookson R. A., 1986; Derivation of Lagrangian and Hermitian shape functions for triangular elements. International Journal of Numerical Engineering, Vol. 23, pp. 275-285.

[14] El-Zafrany A. and Cookson R. A., 1986; Derivation of Lagrangian and Hermitian shape functions for quadrilateral elements. International Journal of Numerical Methods in Engineering, Vol. 23, pp. 1939-1958.

[15] Clint M. and Jennings A., 1970; The evaluation of eigenvalues by simultaneous iterations. Computer Journal, Vol. 13, pp. 76-80.

[16] Corr R. B. and Jennings A., 1976; A simultaneous iteration algorithm for symmetric eigenvalue problem. International Journal of Numerical Methods in Engineering, Vol. 10, pp. 647-663.

[17] Bathe K. J. and Wilson E. L., 1976; Numerical methods in finite element analysis. Prentice Hall Englewood Cliffs, New Jersey. 